\title{
Síndrome de Leriche: revisión de un caso con correlación por angiografía por tomografía computada
}

\section{Leriche's Syndrome: Review of a Case with Correlation in Computed Tomography Angiography}

\author{
M. F. Tapia ${ }^{1,2}$ S. Urbina ${ }^{3} \quad$ T. Elórtegui $^{2} \quad$ G. Schiappacasse ${ }^{1,3}$
}

${ }^{1}$ Hospital Padre Hurtado, Santiago, Chile

2 Universidad Diego Portales, Santiago, Chile

${ }^{3}$ Clínica Alemana Santiago-Temuco, Chile

Rev Argent Radiol 2018;82:39-41.
Address for correspondence María Fernanda Tapia, Residente de Radiología, Facultad de Medicina Clínica Alemana - Universidad del Desarrollo, Las Lilas 651, Rancagua, Chile. Código postal: 2841149, Hospital Padre Hurtado, Santiago, Chile

(e-mail: mariafern.tc@gmail.com).

Estimados Editores,

La enfermedad aortoilíaca oclusiva es una patología que, en su mayoría, es secundaria a una oclusión aterosclerótica de la aorta en su segmento infrarrenal. Se denomina síndrome de Leriche a la oclusión total a nivel de la bifurcación aórtica. Puede llegar a ocasionar una incapacidad para mantener erección peneana, claudicación intermitente junto con disminución o ausencia de ambos pulsos femorales con palidez o enfriamiento y fatiga de ambas extremidades inferiores.

La enfermedad aortoilíaca oclusiva fue descrita por primera vez en 1814 por el británico Robert Graham. Posteriormente fue nombrado síndrome de Leriche, en honor del cirujano francés René Leriche, que realizó un procedimiento quirúrgico en 1948 a un conductor de tractores de 29 años que presentaba ese diagnóstico. ${ }^{1}$

La literatura describe que al menos la mitad de los pacientes con enfermedad arterial periférica no presentan síntoma alguno, por lo que la incidencia real es una condición desconocida. ${ }^{2}$ Sin embargo, se sabe que la incidencia de la enfermedad arterial periférica aumenta con la edad, por lo tanto, se estima que a los 70 años al menos un $25 \%$ de la población de EEUU se encontrará afectada. Ante el síndrome de Leriche el diagnóstico imagenológico cobra gran importancia, incluso por sobre el clínico, debido a la ambigüedad y muchas veces escasez de los síntomas. ${ }^{3}$

Existen tres tipos de compromiso dentro de la enfermedad aortoilíaca oclusiva ${ }^{4}$ (- Fig. 1).

received June 30, 2016 accepted August 1, 2017 published online March 23, 2018
DOI https://doi.org/ 10.1055/s-0038-1637033. ISSN 1852-9992.
El método de imágenes de referencia descrito para el diagnóstico del síndrome de Leriche es la aortografía, ${ }^{4}$ ya que muestra estenosis a nivel de la bifurcación aórtica, calcificaciones y colaterales generadas por la oclusión crónica. La mayor ventaja de esa técnica son sus posibilidades diagnóstico-terapéuticas, siendo posible realizar una terapia endovascular en el mismo acto. Sin embargo, la angiografía por tomografía computada (angiografía por TC) ha desplazado a la aortografía, debido a su gran potencial de evaluación diagnóstica; al mismo tiempo, es un estudio no invasivo que logra disminuir las tasas de complicaciones. La angiografía por TC en pacientes que sufren del síndrome de Leriche puede resultar de gran utilidad.

Presentamos el caso clínico de una mujer de 62 años, hipertensa, fumadora, con un antecedente de cáncer de colon manejado con quimioterapia. Realizó la consulta por una historia de parestesias de extremidades inferiores y una claudicación al caminar que empeoró en los 10 días previos, sin otro síntoma asociado. Se solicitó una angiografía por TC de abdomen y pelvis, cuyo diagnóstico fue un síndrome de Leriche tipo I, ya que en el segmento aórtico infrarrenal, $8 \mathrm{~mm}$ bajo el origen de la arteria mesentérica superior, muestra una alteración en extensión craneocaudal de un segmento de $12 \mathrm{~cm}$, con un diámetro anteroposterior de $36 \mathrm{~mm}$ y transverso de $34 \mathrm{~mm}$. Las arterias ilíacas comunes se encuentran obstruidas desde su origen. Existe un flujo filiforme por arterias ilíacas internas, las cuales reperfunden por colaterales iliolumbares (-Figs. 2-4). Se decidió realizarle un bypass axilofemoral.
Copyright @ $\odot 2019$, Sociedad Argentina de Radiología. Publicado por Thieme Revinter Publicações Ltda., Rio de Janeiro, Brazil. Todos los derechos reservados.
License terms

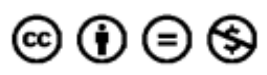



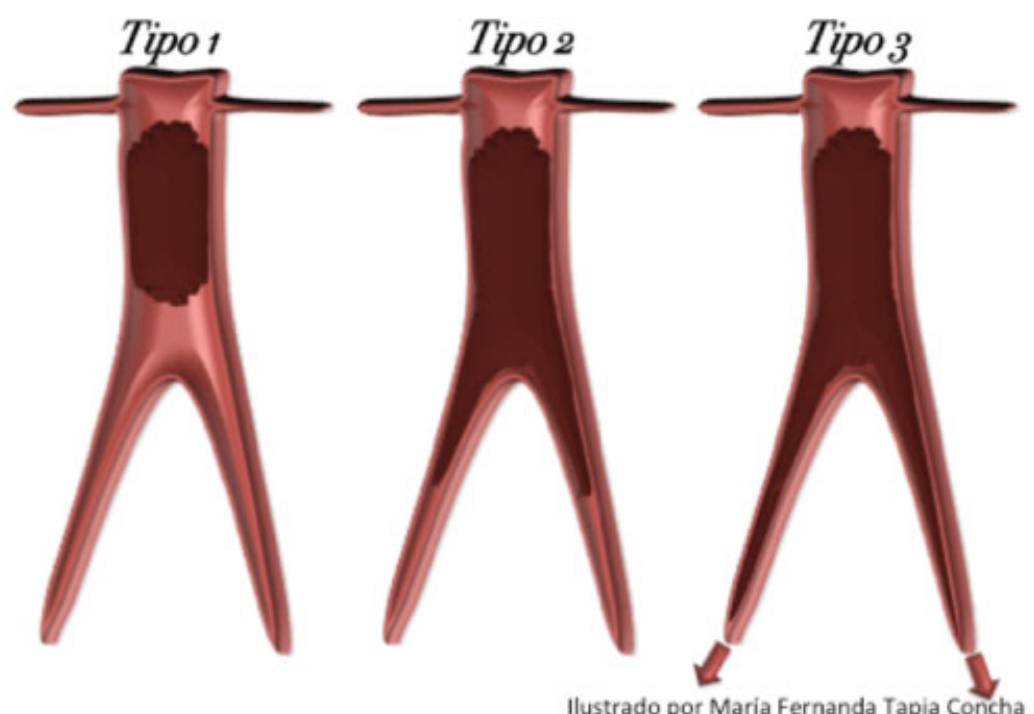

Tipo 1

Compromete aorta

infrarrenal e iliaca común, presente en un $5-10 \%$ de la enfermedad arterial periférica y ocurre más comúnmente en mujeres.
Tipo 2

Compromete aorta

infrarrenal, iliaca común y

externas, puede extenderse

hasta la arteria femoral

común. Se ve en $35 \%$ de los

pacientes con enfermedad

arterial periférica
Tipo 3

\section{Compromete aorta}

infrarrenal, iliacas, femoral, poplítea y tibiales. Forma

más severa y

desafortunadamente más

común.

Fig. 1 Clasificación del síndrome de Leriche.

Es de gran importancia el reconocimiento de signos y síntomas característicos en la historia clínica, para ello, se puede preguntar de manera dirigida acerca de los mismos, especialmente en pacientes con factores de riesgo que tengan una predisposición a esa enfermedad. Lo más importante en el manejo del síndrome es realizar un cambio en el estilo de vida, con una alimentación sana y actividad física, para manejar con anterioridad los factores de riesgo y así evitar los depósitos de placas de colesterol a nivel arterial.

En nuestro caso clínico se utilizó la angiografía por TC como herramienta diagnóstica para objetivar el nivel y grado de oclusión de la aorta y sus ramas. Permitió también su clasificación adecuada y la evaluación de las vías colaterales más frecuentes, siendo ellas la arteria epigástrica inferior,

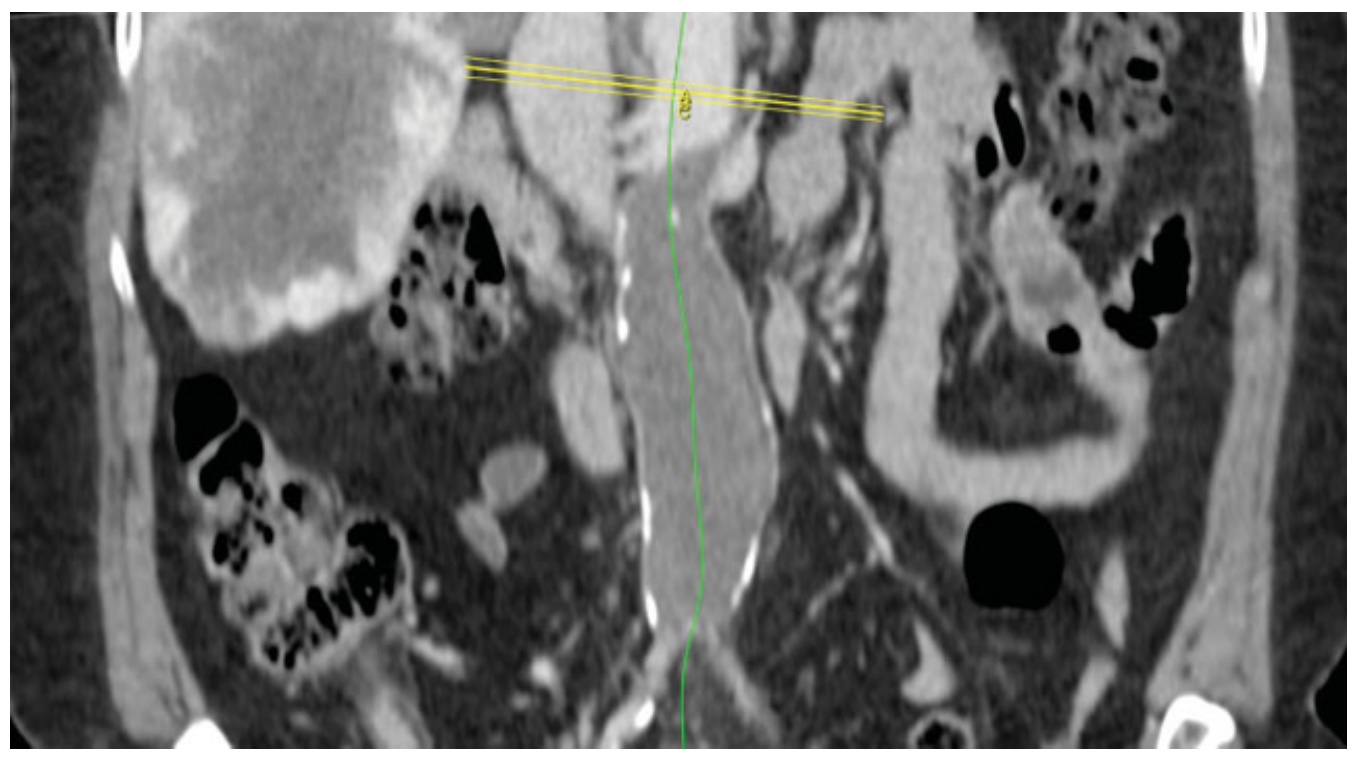

Fig. 2 Angiografía por TC de abdomen y pelvis, MPR coronal de aorta abdominal infrarrenal. Síndrome de Leriche Tipo 1. Se observa extenso trombo en aorta abdominal, $8 \mathrm{~mm}$ bajo el origen de la arteria mesentérica superior con extensión craneocaudal en un segmento de $12 \mathrm{~cm}$, con un diámetro anteroposterior de $36 \mathrm{~mm}$ y transverso de $34 \mathrm{~mm}$. 


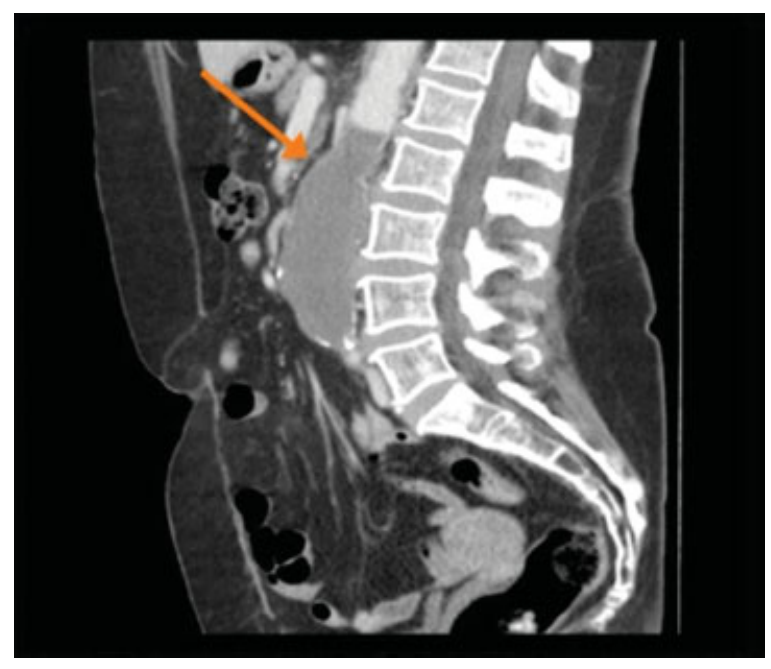

Fig. 3 Angiografía por TC de abdomen y pelvis, reconstrucción sagital. Se visualiza trombo oclusivo de la aorta abdominal, que no realza al contraste, asociado a placas calcificadas en su pared.

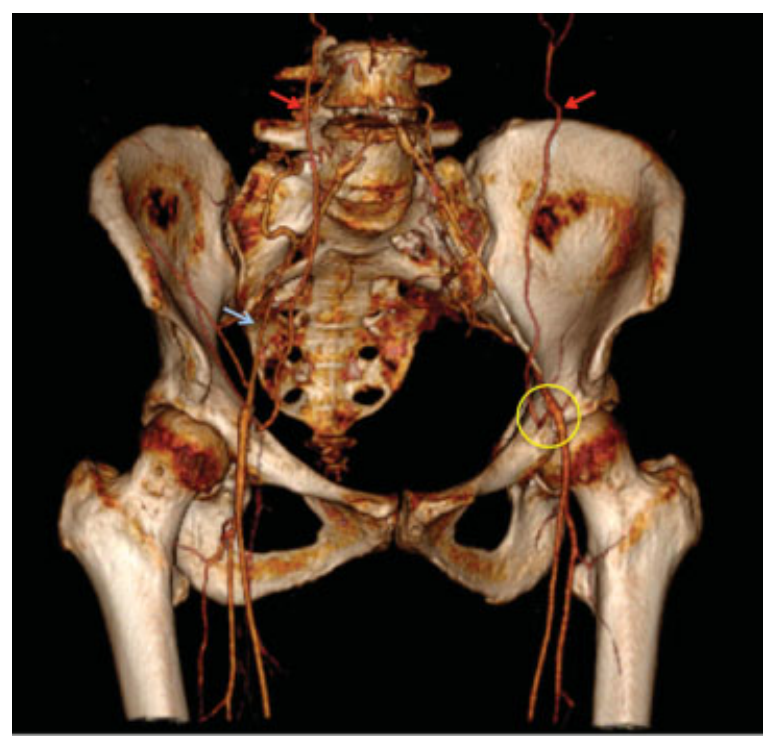

Fig. 4 Angiografía por TC de abdomen y pelvis, con técnica de volume rendering. Se visualiza la irrigación a nivel de la pelvis, con flujo filiforme de arterias ilíacas (flecha azul). Arterias epigástricas inferiorescolaterales (flechas rojas) se anastomosan con arteria iliaca externa a través de la arteria epigástrica superficial (círculo amarillo), restableciendo el flujo femoral. lumbar, intercostal, ilíaca circunfleja profunda, torácica interna y obturadoras, todas derivadas del segmento embriológico de la aorta dorsal. ${ }^{3}$ También pudo observarse el compromiso de órganos a través de la perfusión demostrada con el contraste. Ese test nos permite obtener una imagen diagnóstica sin necesidad de un estudio invasivo, lo que conlleva a una disminución del costo y de las complicaciones a las que podrían estar expuestos los pacientes, brindando una sensibilidad y especificidad diagnóstica muy similar a la aortografía. Permite también la evaluación para la eventual planificación quirúrgica de esos pacientes y, al mismo tiempo, se pueden prevenir lesiones vasculares durante el procedimiento. Además, representa una mejora sobre la aortografía, ya que puede describir compresiones extrínsecas de estructuras vecinas, además de permitir descartar otros diagnósticos diferenciales como la vasculitis. Por lo tanto, pudimos observar que esa técnica imagenológica, es de gran utilidad para el estudio integral de pacientes con síndrome de Leriche y de sus diagnósticos diferenciales.

Los saludan a ustedes, con distinguida consideración, los autores.

Confidencialidad de los datos

Los autores declaran que han seguido los protocolos de su centro de trabajo sobre la publicación de datos de pacientes y que todos los pacientes incluidos en el estudio han recibido información suficiente y han dado su consentimiento informado por escrito.

Conflicto de Intereses

Los autores del trabajo declaran no tener ningún conflicto de intereses.

\section{Bibliografía}

1 Rivera J, Gómez J. Síndrome de Leriche: informe de un caso y revisión de la literatura. Rev Med Hondur 1962;30(sección E)

2 Sebastià C, Quiroga S, Boyé R, Perez-Lafuente M, Castellà E, AlvarezCastells A. Aortic stenosis: spectrum of diseases depicted at multisection CT. Radiographics 2003;23(Spec No):S79-S91

3 Hardman RL, Lopera JE, Cardan RA, Trimmer CK, Josephs SC. Common and rare collateral pathways in aortoiliac occlusive disease: a pictorial essay. AJR Am J Roentgenol 2011;197(03):W519-24

4 Wooten C, Hayat M, du Plessis M, et al. Anatomical significance in aortoiliac occlusive disease. Clin Anat 2014;27(08):1264-1274 